\title{
Cancer Cell Growth - A Mini Review Part-3: Nucleus, PKM2, EGFR
}

\author{
Koyunoglu $\mathrm{C}^{*}$ \\ Department of Chemical Engineering, Energy Institute, Istanbul Technical University, Istanbul, 34469, Turkey
}

\begin{abstract}
The objective of this work is to address the mechanism how cancer cell growth. In a comparable vein, lymphoma preferentially subsets utilize, and can be extremely depending on, oxidative metabolic process instead of the more classical glycolytic phenotype. Hence, lessons to regard how the cell cycle works have been drawn from a wide spectrum of multicellular and single cell organisms arranging from, yeast to plants, to sea urchins to clams, to frogs to mammalian cells. Screens of high-throughput exposed that cells constantly exposed to low glucose need phosphorylation of oxidative as a means preserving growth. To sum up, there is a strong bias of several factors address to cancer growth are summarized in this study.
\end{abstract}

Keywords: Cancer cells; Glycolysis; Warburg effect; Epidermal growth

\section{INTRODUCTION}

When oxygen is plentiful, cells of quiescent effectively draw out energy from glucose mainly by phosphorylation of oxidative, while under the exact similar conditions tumour cells more avidly taken in glucose so as transforming it to lactate. The phenomenon of longobserved is very important for cell development, and is understood as aerobic glycolysis. Since aerobic glycolysis is just beneficial useful for growing cells, it is firmly controlled in a proliferation-linked manner. In mammals, this is partially attained through control of pyruvate kinase isoform expression. The embryonic pyruvate kinase isoform, PKM2, is practically generally re-expressed in cancer, and promotes aerobic glycolysis, whereas the adult isoform, PKM1, promotes oxidative phosphorylation. These two isoforms arise from equally unique alternative splicing of the PKM pre-mRNA, showing addition of either exon 9 (PKM1) or exon 10 (PKM2) [1].

\section{METHODS}

The elements of cell development such as Nucleus, PKM2, EGFR, are summed up gathered from the information of research study works.

\section{Nucleus}

It has actually been reported that no matter how the STAT3 (sense 5'-CACAAUCUACGAAGAA UCAAG-3', antisense 5'-UGAUUCUUCGUAGAUUGUGCU-3') signalling path is triggered, once it translocates to the nucleus, it can control the transcription of its target genes. The information suggested that the translocation of pyruvate kinase type M2 (PKM2) to the nucleus was improved which the nuclear STAT3 and total STAT3 protein levels rose while cytoplasmic STAT3 levels continued to be the same in PKM2 overexpression cells. Remarkably, our information showed that PKM2 localizes to the cell nucleus in addition to living in the cytoplasm which PKM2 over expression upregulates nuclear STAT3 levels, especially phosphorylated STAT3 [2].

In medical practice, breast cancers are graded microscopically on the basis of mitotic counts, pleomorphism of cancer cell nuclei and degree of tubule development, which are then gathered into a total grade rating [3].

Recent studies have shown that $76 \%$ of the human genome is copied to produce a series of lncRNAs. LncRNAs are RNAs of 200 nucleotides (nt) lengths, mostly copied with RNA polymerase II, with abundant structural characteristics of mRNAs consisting of a poly (A) tail, 5 RNA-cap and a supporting structure. Several investigators have also shown that lncRNAs do not have the capacity to protein coding and may be limited to the core and cytoplasm [4].

\section{PKM2}

The pyruvate kinase type M2 (PKM2) is an isoenzyme existing in numerous tissues and multiplying cells. It has actually been reported that cancer metabolic process is associated with the transcriptional activation of PKM2. Specifically, enhancing the ratio of the tetramer and dimer of PKM2 promotes the conversion of glucose to pyruvate for energy production, however reducing the ratio causes the synthesis of nucleic acid, phospholipid and amino acid. At the early stage of tumorigenesis, the tumor cells revealing

${ }^{*}$ Correspondence to: Koyunoglu C, Department of Chemical Engineering, Energy Institute, Istanbul Technical University, Istanbul, 34469, Turkey, Tel: +90(212)2853941; E-mail: ckoyunoglu@itu.edu.tr

Received: January 22, 2019; Accepted: March 25, 2019; Published: April 01, 2019

Citation: Koyunoglu C (2019) Cancer Cell Growth - A Mini Review Part-3: Nucleus, PKM2, EGFR. Biochem Anal Biochem 8:378. doi: 10.35248/21611009.19.8.378.

Copyright: (C) 2019 Koyunoglu C. This is an open-access article distributed under the terms of the Creative Commons Attribution License, which permits unrestricted use, distribution, and reproduction in any medium, provided the original author and source are credited. 
the dimeric PKM2 are generally signified by skin development factor receptor (EGFR), so targeting EGFR can effectively manage cancer cell proliferation [5].

To collaborate glycolysis, glutaminolysis and serinolysis, multiplying cells reveal a specific isoenzyme of pyruvate kinase, described to as pyruvate kinase type M2 (M2-PK). M2-PK happens in a tetrameric kind with a high affinity for its substrate phosphoenolpyruvate (PEP) and in a dimeric type with a low PEP affinity [6].

Finally, pyruvate kinase $(\mathrm{PK})$ plays a critical role in tumor cell metabolism and proliferation as the last rate limiting step enzyme in the glycolytic pathway. Increased expression of Pyruvat kinase type M2 (PKM2) is one of the major causes of increased aerobic glycolysis in cancer cells. Oleanolic acid (OA) found in various diets and medicinal plants has shown that the transition from PKM2 to PKM1, which inhibits the Warburg effect in cancer cells and reduces tumor growth, has been shown [7].

Although PKM2 has lower activity than PKM1, it plays a critical role in fueling cancer cells with intermediates for anabolic reactions. Protein synthesis, an activator of PKM2, is used to synthesize cells for the synthesis of other amino acids (glycine and cysteine), nucleotides (via serine-derived glycine) and the synthesis of lipids such as phosphatidylserine and sphingolipids. In another study, it has been shown that the reduction of pyruvate kinase activity in PKM2 expressing cells leads to the accumulation of 3-phosphoglycerate, which can then be converted into serine, thereby promoting the biosynthetic processes required for cancer cell growth. Synthetic derivatives of OA, especially bardoxolone methyl, have been subjected to phase I clinical trials for cancer patients and have shown promising results [7].

\section{EGFR}

Epidermal growth factor receptor (EGFR) is a trans membrane glycoprotein that is turned on by the binding of EGF and TGF-a on its external domain. After activation, intracellular tyrosine kinases are phosphorylated leading to cell proliferation, change and department. Raised levels of the EGFR and its ligands have actually been determined as a typical element of various cancer types consisting of breast, ovarian, non-little cell lung, colorectal, head and neck cancers. In a lot of cases, aberrant EGFR activation, moderated mainly through modifications in gene amplification and autocrine stimulation, seems a crucial consider tumorigenesis and a driving force for the aggressive development behaviour of cancer cells [8].

While EGFR disregulation is understood to play a vital function in prostate carcinogenesis, there has actually been no direct proof showing EGFR anomalies cause tumorigenesis in prostate cancer [9].

In order to promote efforts to develop new cancer therapies targeting EGFR, monoclonal antibodies and tyrosine kinase inhibitors (TKIs) that target EGFR have to be well studied and promising significant success. For this purpose, many MoAb and TKI compounds targeting EGFR have been studied, and some of these targeting strategies have been tested for safety in target tumors. Clinical trials are currently underway to test the efficacy of these agents [10].

\section{RESULTS AND DISCUSSION}

Here are some researches show the reason or the result of the related topic above mentioned as ordered.
It was determined and established 6-Phosphogluconate dehydrogenase (6PGD) inhibitors, physcion and its acquired S3, that efficiently hindereds 6PGD, cancer cell proliferation and tumour development in naked mice xenografts without evident toxicity, recommending that 6PGD could be an anticancer target [11].

> Tripartite motif-containing protein 35 (TRIM35) belongs to RBCC family, which has actually an extremely saved order including a RING domain followed by a couple of B-Box domains and after that a coiled-coil domain. We formerly determined TRIM35 as a unique tumor suppressor in human hepatocellular cancer (HCC). Nevertheless, the molecular system that TRIM35 utilizes to reduce tumorigenicity is mainly unidentified. Pyruvate kinase isoform M2 (PKM2) has been shown to have a main function in metabolic reprogramming throughout cancer development. Phosphorylation of PKM2 tyrosine residue 105 (Y105) controls PKM2 to offer a metabolic benefit to tumor cells, therefore promoting tumor development [12].

The architecture of cell nuclei is frequently modified in cancer cells. Certain tumour types are connected with particular modifications, and these supply an essential diagnostic function [13].

DRBB receptor tyrosine kinases have vital functions in human cancer. In particular, the expression or activation of skin development aspect receptor and ERBB2 are changed in numerous epithelial tumours, and medical studies suggest that they have essential functions in tumour aetiology and development. Appropriately, these receptors have actually been extremely studied to comprehend their significance in cancer biology and as restorative targets, and lots of ERBB inhibitors are now utilized in the clinic [14].

Pyruvate kinase M2 (PKM2) is revealed at high levels throughout embryonic advancement and tumour development and is very important for cell development. Nevertheless, it is not known whether it straight manages cell division [15].

\section{CONCLUSION}

There are lots of factors that affect basically cancer cell growth since it has to be simplified by using some simplification models as well.

\section{ACKNOWLEDGEMENTS}

Thanks to my mother who encouraged me.

\section{REFERENCES}

1. David CJ, Chen M, Assanah M, Canoll P, Manley JL. HnRNP proteins controlled by c-Myc deregulate pyruvate kinase mRNA splicing in cancer. Nature. 2010;463:364-368.

2. Yang P, Li Z, Fu R, Wu H, Li Z. Pyruvate kinase M2 facilitates colon cancer cell migration via the modulation of STAT3 signalling. Cell Signal. 2014;26:1853-1862.

3. Stephens PJ, Tarpey PS, Davies H, Van Loo P, Greenman C, Wedge $\mathrm{DC}$, et al. The landscape of cancer genes and mutational processes in breast cancer. Nature. 2012;486:400-404

4. Aalijahan H, Ghorbian S. Long non-coding RNAs and cervical cancer. Exp Mol Pathol. 2018;106:7-16.

5. Zhou L, Xu N, Sun Y, Liu X Targeted biopharmaceuticals for cancer treatment. Cancer Lett. 2014;352:145-151. 
6. Mazurek S, Zwerschke W, Jansen-Dürr P, Eigenbrodt E. Metabolic cooperation between different oncogenes during cell transformation: interaction between activated ras and HPV-16 E7. Oncogene. 2001;20:6891-6898.

7. Guerra F, Arbini AA, Moro L. Mitochondria and cancer chemoresistance. Biochimica et Biophysica Acta (BBA) - Bioenergetics. 2017;1858:686-699.

8. Kausch I, Böhle A. Molecular Aspects of Bladder Cancer: III. Prognostic Markers of Bladder Cancer. Eur Urol. 2002;41:15-29.

9. Cai CQ, Peng Y, Buckley MT, Wei J, Chen F, Liebes L, et al. Epidermal growth factor receptor activation in prostate cancer by three novel missense mutations. Oncogene. 2008;27:3201-3210.

10. Wadler S. Targeted Therapy in Colorectal Cancer. Clinical Colorectal Cancer. 2007;6:357-361.
11. Lin R, Elf S, Shan C, Kang HB, Ji Q, Zhou L, et al. 6-Phosphogluconate dehydrogenase links oxidative PPP, lipogenesis and tumour growth by inhibiting LKB1-AMPK signalling. Nat Cell Biol. 2015;17:1484-1496.

12.Chen Z, Wang Z, Guo W, Zhang Z, Zhao F, Zhao Y, et al. TRIM35 Interacts with pyruvate kinase isoform $\mathrm{M} 2$ to suppress the Warburg effect and tumorigenicity in hepatocellular carcinoma. Oncogene. 2015;34:3946-3956.

13.Zink D, Fischer AH, Nickerson JA. Nuclear structure in cancer cells. Nat Rev Cancer. 2004;4:677-687.

14. Hynes NE, Lane HA. ERBB receptors and cancer: the complexity of targeted inhibitors. Nat Rev Cancer. 2005;5:341-354.

15.Jiang Y, Wang Y, Wang T, Hawke DH, Zheng Y, Li X, et al. PKM2 phosphorylates MLC2 and regulates cytokinesis of tumour cells. Nat Commun. 2014;5:5566. 